\title{
ÉTICA Y EPISTEMOLOGÍA EN LA INVESTIGACIÓN CIENTÍFICA. TENDENCIAS Y PERSPECTIVAS
}

\author{
ETHICS AND EPISTEMOLOGY IN SCIENTIFIC RESEARCH. \\ TRENDS AND PERSPECTIVES \\ Ruth Mirihan Rom ero Huam ani* ${ }^{*}$ \\ Docente Auxiliar de la Facultad de Ciencias Contables \\ Universidad Nacional Mayor de San Marcos-UNMSM / Lima-Perú \\ [Recepción: Agosto 2016/ Conformidad: Setiembre 2016]
}

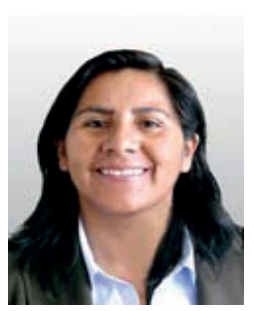

\section{RESUMEN}

La presente investigación es un estudio de carácter cualitativo, es tipo descriptivo y documental, y su propósito es analizar y describir las tendencias y perspectivas éticas y epistemológicas en la investigación científica. La ética y epistemología en la investigación científica es una necesidad básica y fundamental para la ciencia debido a su dimensión filosófica. La ética es una disciplina teórica de la filosofía estudia los principios que regulan el comportamiento y la conducta del ser humano, y la epistemología estudia los procesos y resultados de la investigación científica, ambas son consideradas como observadores de la construcción de la ciencia, es decir la validez o invalidez de la investigación. La posición de la ética en la investigación es un requisito que señala el cumplimiento de la rigurosidad, honestidad, formalización y análisis del campo de estudio. Esto significa que los científicos deben cumplir con la responsabilidad, y objetividad de las consultas teóricas para la obtención de los resultados. Y la posición de la epistemología es ver la coherencia lógica de los procesos y resultados de la investigación. Es considerada ciencia de las ciencias. Asimismo, las tendencias y perspectivas éticas y epistemológicas permiten desarrollar con rigurosidad la investigación.

\section{Palabras Clave:}

Ética, epistemología, investigación científica, enfoque cualitativo, cuantitativo, tendencias y perspectivas.

\begin{abstract}
This research is a qualitative study, descriptive and documentary, and its purpose is to analyze and describe the ethical and epistemological trends and perspectives in scientific research. Ethics and epistemology in scientific research is a basic and fundamental need for science because of its philosophical dimension. Ethics is a theoretical discipline of philosophy that studies the principles that regulate the behavior and conduct of the human being, and epistemology studies the processes and results of scientific research. They are both considered as observers of the construction of science, meaning the validity or invalidity of the investigation. The position of ethics in research is a requirement that indicates compliance with the rigor, honesty, formalization and analysis of the field of study. This means that scientists must fulfill the responsibility, and objectivity of the theoretical consultations for obtaining the results. And the position of epistemology is to observe the logical coherence of the processes and results of research. It is considered science of the sciences. Likewise, ethical and epistemological trends and perspectives allow the rigorous development of research.
\end{abstract}

\section{Keywords:}

Ethics, epistemology, scientific research, qualitative, quantitative, trends and perspectives.

\footnotetext{
* Maestra en Filosofía en Universidad Iberoamericana de México, D. F., Docente de Metodología de Investigación y Tesis en la Facultad de Ciencias Contables- UNMSM. E-mail: rromeroh@unmsm.edu.pe
} 


\section{INTRODUCCIÓN}

La ética y la epistemología en el campo de la investigación científica constituyen un campo de conocimiento y práctica aún compleja. Esta nos remite a plantear la siguiente cuestión: ¿Por qué es importante considerar estas disciplinas para la construcción de una ciencia? Identificar cada una de ellas, dentro de la filosofía como ejes esenciales para la investigación científica y su utilidad en el mundo contemporáneo, es conocer la actividad científica de los profesionales, académicos e intelectuales. Así se van constituyéndo una vía utilizada por la ciencia en cuanto se refiere a la producción de nuevos conocimientos. Por ello, la investigación tiene un sentido lógico cuando cumple los requisitos del método científico y un valor de alto nivel intelectual.

Esto explica que la racionalidad humana es la que ha permitido crear nuevos patrones de la ciencia basada en las preguntas filosóficas desde muchos siglos; cuando el hombre comenzó a cuestionarse del porque de todo, ésta no solamente formó parte de la vida griega sino también en otras culturas ancestrales del mundo. Es por ello, que la investigación es indispensable en todos los saberes humanos y se materializa en los asuntos académicos.

La línea científica basada en las perspectivas y tendencias éticas y epistémicas, en donde tiene su origen, que de manera sistemática determina la lógica de la investigación de los enfoques como: cualitativa y cuantitativa. Estas tienen un origen genealógico desde el punto de vista filosófico y científico.

En los últimos tiempos el papel de la ética y de epistemología ha sido considerado como requisito para el proceso y desarrollo de la investigación científica. Los profesionales de diversas ciencias requieren identificarse con las tendencias y perspectivas de estas disciplinas para demostrar los resultados bajo los principios éticos y epistémicos.

Las bases teóricas de la investigación científica están fundamentadas por los aportes de Platón, Aristóteles, San Agustín, Santo tomas de Aquino, René Descartes, Francis Bacón, Galileo Galilei, Leibniz, David Hume, Emmanuel Kant, G. Hegel, C. Mark, A. Comte, L. Wittgentein, S. Mill, C. Popper, G. Bachelard, T. Khun, I. Lackatos, M. Bunge, F. Kapra, E. Morin, y entre otros; asimismo, las teorías éticas apli- cada a la investigación en el plano contemporáneo comienza en la segunda guerra mundialista se aprecia a los aportes de A Cortina, J. Rawls Ch. Taylor, y entre otros. No obstante, las investigación de Vieira: 2009; Padrón, 2007, Ospina, 2001, Gaudlits, 2008, y entre otros, nos han permitido comprender la funcionalidad de la ética y epistemología en la investigación de manera específica.

En nuestra realidad uno de los focos de significancia son las leyes que direccionan la universidad teniendo como principio la investigación y la profesión como herramientas para la solución de los problemas del Estado, lo cual no se ha logrado aún; es por ello, que la nueva ley universitaria $\mathrm{N}^{\circ} 30220$, señala que la investigación es uno de las bases de la calidad académica y científica.

La ética es la disciplina de la filosofía que estudia los principios que regulan la acción moral del ser humano. La epistemología, también disciplina de la filosofía, estudia la investigación científica y su resultado, el conocimiento científico (Bunge, 2006:14). La ética en la investigación tiene que ver con la relación que tienen una con la otra. Esta relación protagoniza al investigador y su implicancia en la comunidad científica. La persona ética corre el riesgo de olvidar los valores morales en el proceso de la investigación, este olvido no debe realizarse porque el carácter de la ciencia es búsqueda de la verdad (Tamayo, 2004: 207). La relación de la ética, ciencia e investigación se debe a estos criterios: la búsqueda de la verdad, honestidad, bienestar de la comunidad y su entorno, ética profesional, justicia y humildad, secreto profesional (ibíd.)

El conocimiento se refiere a los hechos (...), la moral está conformada por normas y valores para la evaluación de las acciones. Dada la conocida distinción hecho-valor, así como la imposibilidad de derivar lógicamente enunciados evaluativos a partir de proposiciones acerca de hechos, la epistemología... poco o nada tiene que ver ni que ayudar a la ética(Olive, 2007: 85), por tanto, los problemas éticos sí incide en los asuntos epistémicos porque son indispensables a las éticas aplicadas como la bioética por ejemplo.

El objetivo es analizar y describir la ética y epistemología en la investigación científica, mediante la técnica de análisis de contenido, e identificar las ten- 
dencias y perspectivas para determinar los principios éticos y epistémicos en la regulación de las acciones morales del investigador en el proceso del desarrollo de la ciencia.

\section{METODOLOGÍA Y TÉCNICAS}

La presente investigación es de carácter teórico porque se revisan textos que explican las tendencias y perspectivas más representativas y se extrae argumentos para comprender e interpretar. Esto corresponde al enfoque cualitativo y tipo descriptivo y documental, su propósito es analizar y describir la ética y epistemológica en la investigación científica. No presenta población ni muestreo porque se utiliza la técnica de análisis de contenido y el instrumento las fichas bibliográficas para recoger argumentos, citas, resúmenes y otros.

\section{ASPECTOS TEÓRICOS EN EL PROCESO PARACRÓNICO CIENTÍFICO}

La ética y epistemología son disciplinas de la filosofía principalmente y se dedican a corregir y observar los resultados de la investigación. Ambas disciplinas se han desarrollado de manera teórica en la Grecia antigua por Sócrates, Platón y Aristóteles. Cada uno de estos pensadores clásicos desde su enfoque filosófico le han dado sentido y vida a estos saberes y actualmente su aplicabilidad sigue estando vigente para la construcción de la ciencia multidisciplinaria.(Ramón, 2006:19).

El principio de la veracidad es una virtud que permite generar el conocimiento científico coherente, caso contrario, se le denominará plagio científico. La virtud de un investigador radica en este caso en los logros de los objetivos de su investigación y su contribución en el desarrollo de la sociedad. La ética es un saber que se construye racionalmente utilizando el rigor conceptual y métodos de análisis y explicación propios de la filosofía, sobre cuestiones morales que implican situaciones sociológicas, psicológicas, económicos, científicos y otros (Cortina, A. \& Martínez, E. 2008:10).

En el cimiento teórico de la época clásica la corriente eudaimonista explicaba las leyes de la conducta humana que regulan las buenas acciones y que hacen posible el juicio verdadero como la acción bue- na. Siendo el centro de la ética la virtud. La virtud se alcanza por medio del conocimiento: para obrar bien hay que conocer, y el no conocer es la ignorancia o carencia de conocimiento. La epistemología se explicaba desde dos formas: a) el razonamiento abstracto es capaz de proporcionar un verdadero conocimiento y la contemplación filosófica del mundo de las ideas como el fin supremo de la existencia humana; b) la observación cuidadosa y adhesión a las reglas de la lógica, todo conocimiento deriva de la experiencia, permitiendo el desarrollo del método científico bajo el proceso lógico de la inducción.

En la época de Medioevo, la teoría considerada gira en torno al teologismo (San Agustín y Santo Tomas de Aquino) explican la fundamentación epistemológica generando un obstáculo en la libertad e independencia del pensamiento, considerada como la violencia a la conciencia y figura en contra del pensamiento racional. Colocando al individuo fuera, y peor aún, considerarlo un obstáculo para el desarrollo de la libertad y su independencia de pensamiento, esto será visto en nuestra época como una alteración a la conciencia científica. El conocimiento es el razonamiento profundo, la percepción y experimentación basadas en los métodos racionales que serán determinadas por la Fe en un sistema unificado de creencias. El modelo de ética, entre tanto, estaba enfocado al castigo por las acciones negativas que cometía el individuo (pecado) y la recompensa de la inmortalidad para premiar la virtud. Las virtudes más importantes eran la humildad, la continencia, la benevolencia y la obediencia; la espiritualidad, o la bondad de espíritu, que era indispensable para la moral. Todas las acciones, tanto las buenas como las malas, fueron clasificadas por la Iglesia y se instauró un sistema de penitencia temporal como expiación de los pecados.

La sociedad Moderna, estaba enfocada más a la teoría del Humanismo, donde la episteme se constituía bajo los parámetros de los saberes universales que inspiraban la exactitud y la dominación de los métodos científicos, para comprender la subjetividad humana y sus limitaciones dentro del marco de los paradigmas complejos, desde las corrientes racionalistas, empiristas y humanistas. La ética, entre tanto, versaba en la tesis de la conservación de la vida, el deseo, autonomía bajo los parámetros de la conciencia 
moral (Descartes, Espinoza, Kant, y otros).

Las teorías contemporáneas, momento histórico de la posmodernidad, gira en torno a un paradigma deontológico donde el conocimiento es un proceso que constituye y reconstituye a partir de la comunicación de sujetos a propósito del objeto, cumpliendo una serie de decatupla científica. La ética como principio y como valor entra a una crisis de trasfondo, porque se encuentra enmarcado al trabajo y al ahorro. La vida humana se encuentra determinado por los patrones de consumicus reality, es la sociedad de emociones y no de racionalidades; la espíteme avanza en contextos de transdiciplinariedad y complejidad. La investigación desde las concepciones éticas y epistémicas se apropia de los métodos y de hechos humanos, de situaciones concretas y de teorías sujetos a cambio permanente, de diversidad de conductas humanas abarcando sentimientos, aptitudes, actitudes y todas las facultades humanas dentro de criterios de relatividad.

\section{ÉTICA DE LA INVESTIGACIÓN CIENTÍFICA}

Qué es la ciencia y cuál es su ética. La ciencia es una empresa que se desarrolla de la mejor manera cuando los científicos trabajan de acuerdo a los principios éticos. La ética define la condición humana cuando su preocupación es el comportamiento y carácter de las personas en situaciones específicas y circunstancias particulares(Koepsell\& Ruiz de Chávez: 2015). La discusión en la ciencia actual es la ética, como disciplina determinante convierte a la investigación científica en una buena opción para el desarrollo de la sociedad. La ética en la investigación constituye un conocimiento teórico y práctico que regula la moralidad del investigador desde el momento que idealiza los temas de investigación y la elaboración del marco teórico, cabe señalar que el marco teórico es la perspectiva que permite la construcción y el logro de los objetivos y demostración de las hipótesis en ambos enfoques de la investigación como cualitativa y cuantitativa.

En la comunidad académica - intelectual de la empresa universitaria la responsabilidad moral tiende a ser objeto de cuestionamiento cuando los resultados no muestran la coherencia lógica de la investigación, esto sucede cuando el investigador de diferentes áreas de la ciencia, no posee mayor conciencia de la necesidad de los principios éticos y valores morales como tales, asimismo sobre las perspectivas y tendencias de la ética.

\section{Algunos problemas que generan la debilidad de los criterios éticos}

No resulta fácil controlar éticamente los abordajes cuantitativos de una investigación porque se produce varias debilidades en los investigadores, tales como:

1. Tematización de las ideas de investigación. No es fácil tener ideas sin tener una lectura suficiente de las fuentes de información. Siendo esta la causa de la ausencia de ideas o surgimiento de ideas vagas.

2. Falta de dominio de la navegación científica. Muchos de los que investigan o se están iniciándose no conocen los criterios de la navegación científica.

3. Desconocimiento de las normas para la cita y referencias bibliográficas

4. Desconocimiento de los métodos generales y específicos de la investigación.

5. La proclividad en utilizar sujetos de estudio como elemento de investigación, sin buscar una comunicación para entender el significado de los hechos (Bautista, 2011:22).

6. El uso inadecuado delas técnicas de investigación y los resultados que no son comunicados a los sujetos de estudio.

7. La técnica de entrevista estructurada como limitante para recoger la data por presentar preguntas puntuales.

8. La confianza exagerada en una encuesta como fiable y única para recoger opiniones.

9. La falta de dominio acerca de los tipos y diseños de investigación como esenciales para determinar el muestreo. Etc.

10. Mediante la observación directa se ha percibido en las áreas de posgrado, el desconocimiento de los alumnos sobre la metodología de investigación un $80 \%$ y en pre grado $94 \%$. Siendo esto la causa de la debilidad de producción científica y el desarrollo profesional. 
En cuanto al abordaje de las investigaciones cualitativas es diferente, por los siguientes motivos:

1. La legitimación de las fuentes primarios para comunicar de manera horizontal entre el investigador y los sujetos investigados, generan un tipo de confiabilidad, esto dependerá qué criterios utiliza el sujeto investigador para la selección de la fuentes escritas.

2. La moralidad del investigador se debe a una estrategia más natural ya que su imagen se desmitifica cuando extrae al exterior la capacidad de interpretación de los resultados de la entrevista a profundidad.

3. En el campo de ciencias sociales las conductas éticas del investigador se perciben cuando cosifica a los sujetos de investigación (volverlos cosas), cuando se cree que los problemas sociales dependen de las estructuras económicas y recursos tecnológicos.

4. Cuando se pretende interpretar las normas, leyes, reglamentos y otros, no se utiliza métodos adecuados, por falta de criterios epistemológicos y se deja, solamente, como citas textuales.

5. En las ciencias humanas las interpretaciones muchas veces son inadecuadas, pero esto no implica una falta grave sino son criterios de cada investigador si lo respetan los aspectos hermenéuticos. Para poder evitar las malas prácticas en la investigación es necesario analizar la importancia que tienen las perspectivas y tendencias de la ética, asimismo la funcionalidad de los principios éticos en la producción de las ciencias.

TENDENCIAS DE LA ÉTICA. Son los que más influyen en la investigación científica actualmente.

\section{1. Ética eudaimonica y hedonismo}

Esta tendencia de la ética gira en torno a la virtud y se consigue solamente por medio del conocimiento. Uno de los deberes naturales del hombre es buscar el conocimiento. El hombre es por naturaleza un investigador; por ello, su actuar siempre debe ser bajo la mirada de la acción buena. Cualquier disciplina científica como la política, la cultura, la educación y otros, debe estar bajo la autoridad de la ética, ya que ella es la única que regula las malas acciones. (Aristóteles,citado por Ramón, 2006:23).Toda reflexión humana está orientada a resolver problemas de deferente índole dentro de la convivencia social, ya que el objetivo es cómo alcanzar la felicidad. Todo investigador honesto buscará la felicidad, si investiga bajo los principios éticos. Esto se conseguirá cuando se logra el objetivo de la investigación al finalizar y sustentar la tesis.

\section{2. Ética hedonista}

La cuestión ética del placer consiste en determinar el bien y el mal. Todo tipo de placer se sitúa en la felicidad humana porque el placer es el único bien supremo. El placer en la teorías del hedonismo, es considerada por su extensión, como una utilidad, de ahí se desprende el utilitarismo, mientras el hedonismo como sumo bien del placer, el bienestar y la utilidad social. Esto nos lleva a señalar que el hedonismo tiene dos consecuencias, por un lado tiene un carácter individualista y otro de índole social. La primera en el campo de la ciencia, determina la felicidad individual del investigador, cuando logra su objetivo científico y resuelve los problemas sociales y otros. La segunda está enfocada a la utilidad de los trabajos de investigación en la sociedad, generando así el bien común, como por ejemplo las investigaciones en el campo de salud como los medicamentos que ayudan a mejorar el dolor de las personas.

\section{3. Ética instrumentalista}

Es una teoría filosófica de carácter pragmatista que fue propuesta por Jhon Dewey más o menos en los años veinte del siglo XX. Desde este enfoque el conocimiento es un instrumento de acción con que el hombre se adapta al medio y lo domina. Esta teoría es pragmatista porque afirma que la formación de los conceptos y de las cosas del mundo, forma parte del sometimiento de la prueba para averiguar sus posibles consecuencias. Se afirma que el pensamiento funciona según ciertas reglas que, una vez codificadas, dan lugar a la "lógica de la investigación", porque se sostiene en el criterio utilitarista, esta busca el máximo bienestar del mayor número de individuos, la felicidad general, como criterio y fin de la moralidad, 
apelando al sentido común de los seres humanos para ser tenido como principio y guía de la acción. (Mill, S., 2014) Para esta corriente lo útil genera la felicidad de los individuos. Lo útil es ser práctico, es decir valorar de manera distinta las cosas según el uso.

Ahora bien, en nuestro medio académico lo útil es tener un grado académico o título profesional para ser reconocido como tal en la sociedad, por eso de manera empírica se ha visto que algunas universidades de la última generación de carácter empresarial asociativas o societarias se han dedicado entregar los grados y títulos sin considerar los requisitos especiales que exige obtener los estándares internacionales.

Dentro de este contexto, uno de los requisitos es la investigación; sin embargo, muchas de los egresados de las universidades no tienen suficiente criterio para elaborar una investigación científica. Es por ello, la utilidad instrumental de obtener un título profesional o un grado de manera individual sea malo o bueno no sería útil para el cambio de la sociedad, si solamente se guarda en los archivos de la biblioteca y no es consultado por nadie, y si es fuente de consulta y beneficia a otros investigadores como teoría sustantiva y en su ejecución práctica, entonces su utilidad proporcionaría un bienestar al mayor número de personas investigadores o colectivos.

\section{Humanismo}

La ética del humanismo forma parte de la dignidad y autonomía del individuo como eje esencial.
Su compromiso es maximizar la libertad de elección basada en: Libertad de pensamiento, libertad de conciencia, el libre pensamiento, la libre investigación, y el derecho a estilos de vida de acuerdo a sus posibilidades sin perjudicar a otros. Esta filosofía coexiste en una multiplicidad de sistemas alternativos de valores que aprecian la diversidad social. En una academia, donde se genera investigación para el desarrollo de la sociedad, lo que se acepta por humanismo, es la defensa de la conducta del investigador. Siendo el objetivo el logro de un nivel cualitativo de gusto y distinción. Las libertades como principio ético deben ser aplicadas con responsabilidad por el investigador, ya que se vive en el seno de comunidades científicas. En el contexto humanista la investigación científica es una producción que satisface al investigador y beneficia a los otros individuos, cuando se realiza experimentos y los resultados pueden ser beneficiosos para la humanidad en la cura de una enfermedad por ejemplo.

\section{Dialógica o discursiva}

Consiste en estudiar la moral desde un carácter universalista, autores como de Otto Apel desde los presupuestos universales de la argumentación y de la acción comunicativa como J. Habermas, desarrollan la ética desde dos enfoques: la pragmática trascendental (Apel) y la pragmática universal (Habermas). Y de ahí plantean modelos éticos que serán detallados en la Tabla $\mathrm{N}^{\circ} 01$.

\section{Tabla No 01}

\begin{tabular}{|c|l|l|}
\hline \multirow{4}{*}{$\begin{array}{c}\text { Modelos } \\
\text { éticos }\end{array}$} & $\begin{array}{l}\text { Pragmática } \\
\text { trascenden- } \\
\text { tal }\end{array}$ & $\begin{array}{l}\text { 1. Argumento como punto de partida } \\
\text { 2. Generación del discurso es irrevisable y constitutivo para la reflexión trascendental y convir- } \\
\text { tiéndose en elementos de fundamentales. }\end{array}$ \\
\cline { 2 - 4 } & $\begin{array}{l}\text { Pragmática } \\
\text { universal }\end{array}$ & $\begin{array}{l}\text { 1. Interacción comunicativa como punto de partida } \\
\text { 2. Los elementos que articulan la teoría discursiva son constructos de la reflexión susceptibles de } \\
\text { revisión analítica. }\end{array}$ \\
\hline
\end{tabular}

Fuente: Elaboración propia.

La ética del discurso es considerada como una ética cognitivista porque pretende fundamentar los juicios morales, la universalista fundamenta con juicios morales de validez general, y desde la concepción deontológica las cuestiones de justicia de las normas ante las cuestiones relativas de la "vida buena" y, el formalista regula un procedimiento normativo imparcial de resolución de problemas.
Se convierte en el universalismo la ética discursiva cuando los resultados pretenden superar las limitaciones implícitas en la investigación en los contenidos concretos.

\section{Relativismo}

La ética desde esta concepción es la posición de las normas sociales, y ésta es la que determina la moralidad de las personas. A este relativismo ético 
se le categoriza con ética situacional porque depende de modus operandi en la historicidad del tiempo. Su ventaja radica en ampliar la variedad de hábitos y costumbres de culturas y prácticas. También les permite a las personas adaptarse éticamente a los cambios de la cultura, el conocimiento y la tecnología en la sociedad. Dicha ética se manifiesta en las investigaciones de carácter etnográfico y casuísticas y del diseño longitudinal. Por otro lado, su alcance relativo se manifiesta cuando los resultados de las investigaciones son refutados y cambiados por otras nuevos en el tiempo, esta forma partes de una de las características de la ciencia relativa.

\section{PERSPECTIVAS ÉTICAS EN LA INVESTIGACIÓN}

1. Consecuencialismo. Es una postura mental en los asuntos de la moralidad. Y es una consecuencia de las acciones para juzgar, si las situaciones específicas son buenas o malas. Esta corriente tiene reglas que definen las acciones: Una conducta es buena, si los efectos o consecuencias que tiene son buenos y una conducta es mala, si los efectos o consecuencias que tiene son malos (George \&Tollefsen, 2012). Estas reglas son el fundamento de la ética que explica la realidad empírica desde la búsqueda del placer y la situación del dolor como universales, por tanto los valores como universales e empíricos constituyen la medida del comportamiento ético en las personas.

2. Ética deontológica. La Deontología supera una limitación importante de la teoría ética de la virtud, al proporcionar una guía para la acción, en lugar de concentrarse en mejorar el carácter como señalaba Aristóteles. La ética deontológica,afirma que se deben acatarse ciertos deberes, los cuales se pueden establecer de distintas formas. Se basa en la teoría del derecho, nuestras obligaciones se derivan de nuestra responsabilidad de reconocer y proteger los diversos derechos como la vida, la libertad, la propiedad y propiedad intelectual.

Lo más destacable es la tesis deontológicas, es la del imperativo categórico de Kant (1785): Actúa sólo de manera que quieras que tus acciones se conviertan en una ley universal, aplicable a todas las personas en una situación similar. Actúa de manera que siempre trates a la humanidad (ya sea uno mismo u otro) tanto como medio de una acción, como un fin en sí mismo. Y Actúa como si fueras al mismo tiempo un miembro y rey de un hipotético reino de los fines; $y$, por tanto, sólo de manera tal que la máxima acción armonice con icho reino, como si esas leyes fueran vinculantes para todos.

Con estos imperativos se determina la ética de la investigación y la integridad científica, que para muchos es improbable y para otros se debe a las normas de cada institución que regula acciones trascendentales.

3. Ética de la virtud. La ética de la virtud es la base del bien, Aristóteles señalaba que cultivar un buen carácter, implica desarrollar ciertas virtudes. Platón describió cuatro virtudes cardinales, prudencia, justicia, fortaleza y templanza; y su cultivo sólo podría lograrse a través del estudio adecuado y la moderación de nuestras emociones. Uno de los pioneros contemporáneos de esta ética es Jhon Rawls, (2002) La racionalidad basada en la teoría de La Virtud radica en el incremento del número de virtudes. Es moral, en el sentido de que se busca de forma activa y consciente. Nuestras inclinaciones virtuosas y viciosas están en la introducción a la ética de la investigación y la integridad científica con la finalidad de actuar de conformidad con los principios como lo serían la honestidad, justicia, templanza y prudencia.

\section{PRINCIPIOS ÉTICOS QUE DEFINEN LA ÉTICA DE LA INVESTIGACIÓN}

1. Justicia. Como principio se constata en el riesgo mínimo para el beneficio que se obtendrá y la moralidad de la persona que persigue el placer. La equidad como valor define la vulnerabilidad y la compensación. Cuando asociamos principio de justicia con la investigación científica, podemos aseverar que lo justo se identifica con lo bueno y lo correcto.

2. Libertad. La libertad de elección para la investigación es fundamental, ya que ella es una facultad humana, esta no es sino libertad de expresión en el campo de la investigación científica. La libertad de investigación científica tiene una identificación terminológica y conceptual con la libertad de cátedra, esto sucede en el círculo universitario 
y comunidades científicas, porque la búsqueda del conocimiento y su transmisión van unidas.

3. Autonomía y Beneficencia. El principio de autonomía ocupa un lugar central en el pensamiento humano, porque es la piedra angular para lograr el bien. Para alcanzarse requiere del ejercicio de la razón práctica; esto representarála realización de la dignidad como seres racionales. Esta noción de autonomía moral es muy semejante a lo que podríamos considerar libertad de conciencia, idea y de pensamiento (Kant, A. \& Mill S.). Se trata de la protección y confidencialidad de los datos y la comprobación de los resultados con total objetividad sin causar daño al otro.

4. Alteridad. Es un principio que permite alternar o cambiar la propia perspectiva por la del otro. Por ello, la alteridad implica que un investigador sea capaz de ponerse en el lugar del otro, o sea del fenómeno investigado, lo cual posibilitará establecer relaciones con los otros basadas en el diálogo y la conciencia y valoración de las diferencias existentes. Además, implica constituir una individualidad considerando: la existencia de un colectivo como primero y necesario, es decir, el yo existe a partir del otro y de la visión de este. El otro permite que el yo pueda comprender el mundo a partir de una mirada diferente en relación con la propia.

5. Proporción de riesgo. La obligación por el respeto a la integridad del ser humano y a la dignidad de la personas es fundamental, por tanto la investigación sobre el ser humano, los intereses de la ciencia y de la sociedad nunca podrán prevalecer sobre el bienestar del sujeto como simplicidad. Los experimentos con los sujetos deben cumplir los requisitos de códigos éticos para evitar riesgos o molestias que conlleven a la vida. Todo proyecto de investigación con los seres vivos especialmente con los humanos debe estar precedido de un cuidadoso cálculo de los riesgos t debe pasar por un protocolo del comité científico.

\section{EPISTEMOLOGÍA DE LA INVESTIGACIÓN CIENTÍFICA}

Es un conjunto de razonamientos lógicos y una serie de análisis de datos teóricos e empíricos y su validez y logro se presenta independientemente del sujeto investigador. Para eso es indispensable cumplir requisitos mínimos:

1. Elegibilidad de teóricos sustantivas

2. Elegibilidad de datos empíricos

3. Trasparencia de métodos generales y particulares

4. Explicitación de razonamiento lógico para obtener conclusiones validas

5. Publicación y discusión abierta para el conocimiento de la comunidad científica

La comunicación epistemológica de una investigación científica constituye en la elaboración de programas y la formulación de modelos mediante los cuales se simplifica los conceptos generales de dichos programas.

Tendencias epistemológicas: el objetivo de las tendencias epistemológicas es ayudar a obtener conocimientos válidos, que gocen de solidez científica a través de un proceso racional, sistemático y riguroso en las disciplinas. Por ello, es los últimos tiempos ha surgido tendencias epistemológicas, que has provocado nuevos problemas en el ámbito filosófico, social, cultural, entre otros, a la vez han generado un replanteamiento de cuestiones clásicos, y nuevas propuestas de solución y nuevas vías de búsqueda. Las nuevas vías como tendencia surgen de los variables de objeto, sujeto y la relación de ambos; siendo estas las que crean las siguientes enfoques: empirista-realista sirve para mediciones, experimentaciones y inducción controlada; empirista-idealista para la investigación etnográfica, diseños de convivencia y inducción reflexiva; racionalista-realista para las abstracciones, sistemas lógico-matemáticos, deducción controlada; racionalismo-idealismo para las interpretaciones libres, lenguajes amplios, argumentación reflexiva.De esto surgen epistemologías y son operativas en la producción científica. Aunque la teoría falsacionista señale que nunca se puede hallar la verdad, solo identificar algunos errores. Lo que pretende es que la comprensión de las tendencias que ayude al investigador la verdad científica.

Objetivismo. Es una posición epistemológica que da primacía al objeto en sus relaciones con el sujeto, estas son cognitivas o valorativas. De acuerdo a esta corriente el valor como tipo ideal y como 
objeto. Como tesis filosófica es relativa a la verdad porque se centra en el mundo exterior y se obtiene información a través del método de la observación. Con esto se constata que la verdad es independiente de las personas que la piensan. La tarea del investigador espercibir esa realidad sin inventar ni recrear. Porque rechaza la subjetividad y las creencias del mundo no natural.

Subjetivismo. Es la teoría filosófica y afirma que la realidad se somete al pensamiento, esta tesis es contrario de las tesis del Objetivismo. Porque tiene preferencia especial al carácter de todos los fenómenos de la conciencia, es la introspección del sujeto del conocimiento en el sentido de significancia de las explicaciones de los resultados científicas. Es decir, parte del principio de que las condiciones particulares son las que determinan los juicios formulados en una investigación. Esta corriente es un elemento de gran trascendencia cuando acepta la existencia de intersubjetividades (fenomenológico).

Constructivismo. Esta perspectiva define el debate sobre la integración de los denominados métodos cuantitativos y cualitativos en la investigación científica, dada la complementariedad recíproca que existe entre ellos para abordar integralmente el objeto de estudio, así como por la implicación ontológica que el observador tiene en la construcción de la realidad que describe, comprende y explica (Pérez,2005:9). Además contribuyen a fundamentar cuatro paradigmas como: Teoría sistémica, realidad como construcción de la vida, la mirada sobre la vida cotidiana como privilegio y aceptación del lenguaje generativo.

Holismo. Es una doctrina que explica el todo. En la Grecia antigua se afirmaba que todo era la suma de sus partes, es decir la explicación universal de las cosas. (Aristóteles). El enfoque integral del ser humano se inició desde la ciencia antigua, sin embargo en el transcurrir del tiempo surgió una fragmentación en modelos epistémicos. El holismo es un conocimiento, un procedimiento global, evolutivo, integrador, concatenador y sinérgico, con aspectos secuenciales y simultáneos La ruptura epistémica permitió remodelar la opción ontológica en la investigación. El enfoque holístico en la investigación surge como respuesta a la necesidad integradora de los diversos enfoques, métodos y técnicas, que desde diversas disciplinas científicas han permeado el desarrollo del conocimiento humano. (Hurtado de Barreda, 2002. Citado por Bautista, op. cit.: 36). Esta es una síntesis que integra el corpus metodológico complejo, estructural y sintético, afirma Morín, E. (2000) porque explica de manera más sencilla, práctica y coherente múltiples campos de la ciencia. Además, permite la disolución de falsas y extraviantes relaciones antitéticas, siendo su objetivo el logro sucesivo de un proceso continuo y no resultado final.

Esta propuesta metodológica refleja y contiene las dimensiones de la totalidad; para ello, se debe cumplir la funcionalidad de los principios, como se aprecia en la Tabla $\mathrm{N}^{\circ} 02$.

Tabla No 02

\begin{tabular}{|l|l|}
\hline \multicolumn{1}{|c|}{ PRINCIPIO DE HOLISMO } & \multicolumn{1}{|c|}{ ROL DEL INVESTIGADOR } \\
\hline Principio de continuidad & Presenta una visión universal y aborda desde la evidencia la totalidad. \\
\hline Principio de evolución: la investigación como devenir & $\begin{array}{l}\text { Transcurre en el devenir de la humanidad con el fin de comprender el proceso evolutivo de la historia } \\
\text { de los fenómenos como problemas. }\end{array}$ \\
\hline $\begin{array}{l}\text { Principio holográfico: el evento contiene al todo Princi- } \\
\text { pio del conocimiento fenomenológico: investigador como } \\
\text { ser de situación }\end{array}$ & La totalidad coherente es lo que hace el proceso de asesoría y tutoría de la investigación \\
\hline $\begin{array}{l}\text { Principio del conocimiento fenomenológico: investigador } \\
\text { como ser de situación }\end{array}$ & El investigador como persona contextualiza la situación particular de un problema en sí. \\
\hline $\begin{array}{l}\text { Principio de integralidad: la holopraxis como vivencia } \\
\text { holística }\end{array}$ & $\begin{array}{l}\text { Es una vivencia holística en la cual se integra las dimensiones del ser humana, cuyo objetivo es la } \\
\text { preservación de la vida. }\end{array}$ \\
\hline
\end{tabular}

Fuente: Hurtado de Barreda (2011: 37-38)

Pragmatismo. Esta es una corriente filosófica explica al conocimiento como instrumento de acción y busca criterios de verdad de los teorías en su éxito práctico. Es un método en primer lugar y en segun- do una teoría genética. Otros los conciben como paradigma porque se caracteriza en su influencia a los enfoques de investigación. Un investigador es pragmático cuando demuestra la verdad científica 
basándose en este paradigma y los resultados que se convierten en una acción aplicable (investigación tecnológica).

Perspectivas epistemológicas: la epistemología es el tratado sobre el conocimiento o para algunos es la doctrina del saber. Todo investigador social debe considerar las posturas epistemológicas con la finalidad de direccionar la investigación y para la elaboración de tipos de instrumentos y técnicas para recoger la data. Por ello, la perspectiva de esta disciplina es crear miradas epistémicas valiéndose de las ontológicas para que toda investigación tenga la utilidad paradigmática, es decir, la creación de una teoría, esto significa hacer epistemología de alguna ciencia (objeto, sujeto y la relación de ambos).

Interpretativismo. En el campo de las ciencias sociales los métodos de investigación cualitativa como cuantitativa, tienen una diferencia muy marcada por la ideología histórica. El punto esencial está en lo que se observa y en lo que se interpreta. Esto define los enfoques de la investigación. La interpretación es propia del enfoque de la investigación cualitativa, porque forma parte de las ciencias sociales. A ella se define como acto de dar un determinado sentido a cierta realidad en la que se muestra las intencionalidades, es decir la realidad como objeto de interpretación que hace el sujeto. La interpretación es buscar la comprensión de los fenómenos en diferentes contextos con la finalidad responder a las preguntas como: qué, qué busca, de qué habilidades requiere, cuál es su propio principio de análisis, etc.

Hermenéutica. Es un principio como el arte de interpretar para fijar el verdadero sentido de los fenómenos, que esencialmente servía en algún momento de la historia como interpretación de los escritos sagrados. A partir del siglo XIX se entiende como la reconstrucción de la comprensión de las realidades como es el caso de las emociones subjetivas y sus contextualización. En el ejercicio de la investigación es la indagación sobre el impacto de los hechos y de los actores sociales de manera objetiva. Su aplicación como método o como modelo es para el enfoque cualitativo, porque crea categorías abstractas para teorizar y contextualizar los constructos.

Fenomenología. La fenomenología describe las estructuras de las experiencias tal como se presenta en la conciencia, sin recurrir a la teoría, deducciones o suposiciones precedentes de otras disciplinas como ciencias naturales. Su utilidad está enfocada a fenómenos complejos. El objetivo central es comprende las habilidades, prácticas y experiencias cotidianas y articular las similitudes y las diferencias en los significados, compromisos prácticos, del investigador. Como las preguntas que se puede utilizar para determinar el tema de investigación, partiendo de: qué es, qué busca, de qué habilidades requiere, y cuál es su principio de análisis. Además se considera como método filosófico, porque extrae situaciones esenciales de los fenómenos.

Interaccionismo simbólico. Es la corriente que surge en el siglo XX, y tiene su fundamento en la comunicación de los sujetos relacionados entre sí, los modos y convenciones de la interacción social están regidos por la historia y lugar. Se observan todo un cambio que genera los actores sociales formando acuerdos que afectan de manera recíproca. Esta perspectiva tiene una dimensión metodológica dentro de la ciencia social empírica, porque ofrece un conocimiento de la vida de grupo y el comportamiento humano esta se puede verificar en los resultados de investigación. El investigador interaccionista se dedica e indagar estudios de caso y análisis comparativo apoyándose en los símbolos como en las ciencias antropológicas y otros.

Teoría crítica. Según la escuela de Frankfurt, en esta perspectiva es necesaria la conjunción de los conocimientos de antropología, estética, sociología, artes y la filosofía con la finalidad de recrear la relectura de las teorías para comprender de manera profunda la génesis de dichas teorías. La investigación desde esta perspectiva tiene un horizonte de crear especialidades y para comprender mejor la sociedad como totalidad, para ello se considera las interrogantes como: Qué es, qué busca, de que habilidades requiere y cuáles s su principio de análisis.

Paradigma de la complejidad. Según Morín, E. (2000) es la aceptación de la totalidad, su objeto es estudio es la realidad. La complejidad elimina la simplicidad. Es un tejido de componentes heterogéneos inseparables que presenta la paradoja de lo uno de los múltiple. En las ciencias empresariales es la construcción de nuevos descubrimiento de la estructura de una empresa.

La epistemología compleja es un objeto comple-

52/ QVIPURAMAYOC | Vol. 24(46) 2016 
jo que se explican con dos razones: a. Es complejo el conocimiento porque constituye una totalidad organizada compuesta por un conjunto de elementos heterogéneos (biológicos, sociales, culturales, psicológicos, lógicos, históricos) y en interacción. b. El conocimiento es un objeto complejo puesto que los elementos y procesos que lo constituyen no pueden ser abordados por una disciplina particular. El programa de investigación de la epistemología compleja rompe la equivalencia y la identidad de la epistemología con cualquier disciplina particular.

\section{PROPUESTA APLICATIVA}

Todo hallazgo científico o de divulgación tiene su aplicación práctica. En este caso la operatividad del conocimiento se debe ejecutarse para que toda investigación tenga fundamento ético y epistemológico y de esa manera pueda tener su trascendentalidad. En la Facultad de contabilidad se requiere implementar la enseñanza de la epistemología en pre grado y posgrado con la finalidad de generar una investigación científica, tecnológica y con trasfondo lógico. Las tesis universitarias deben cumplir con los principios éticos y epistemológicos, por ello es requisito indispensable implementar la propuesta que continuación se muestra con la finalidad de mejorar la calidad de las tesis científicas, tal como se detalla en la Figura $\mathrm{N}^{\circ} 01$.

\section{Figura No 01}

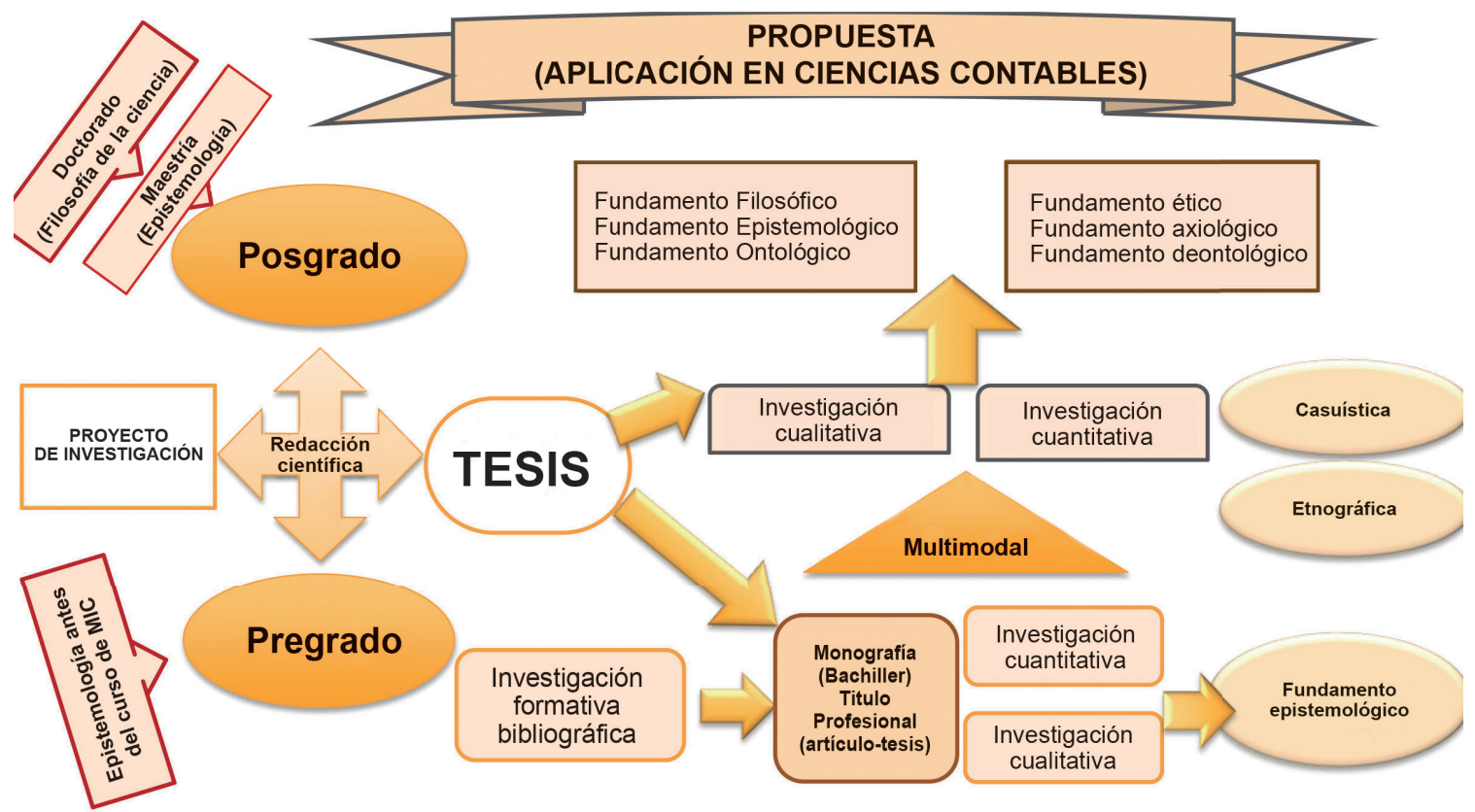

Fuente: Elaboración propia

\section{CONCLUSIONES}

1. La ética y epistemología es fundamental para la construcción de la investigación sea de carácter cualitativa o cuantitativa.

2. Las tendencias y perspectivas de ambas disciplinas regulan las acciones morales y epistemológicas de los resultados de la investigación y el rol del investigador.
3. La investigación científica es una actividad reconocida, que está regida bajo los principios éticos y epistémicos para su fiabilidad y validez de los resultados y su relación con los beneficios que las investigaciones brindan a la sociedad. 


\section{REFERENCIAS BIBLIOGRÁFICAS}

1. ARIAS, S. \& PEÑARANDA, F. (2015). La investigación éticamente reflexionada/ Ethically pondered research/A pesquisa éticamente refletida. Revista De La Facultad Nacional De Salud Pública, 33(3), 444-451. Retrieved from http://search.proquest.com/ docview $/ 1725044510$ ? accountid $=12268$

2. BACHELARD, G. (1973). Epistemología, Barcelona. Editorial Anagrama.

3. BACHELARD, G. (2000). La formación del espíritu científico. Siglo XXI México.

4. BAUTISTA, N. (2011) Proceso de investigación cualitativaepistemología, metodología y aplicaciones. Colombia, Ed. Manual Moderno.

5. BUNGE, M.(2006a) Epistemología. Quinta edición. México D.F., Siglo XXI Editores. Barcelona: Gedisa.

6. BIRKE, A. (2004) Papel de la ética en la investigación científica. México. ed. Lemusa.

7. CARRASQUERO, A.(2014). Investigación holística y Desarrollo Instruccional en la comprensión del discurso escrito en estudiantes de educación media de la U.E.N. José Félix Blanco. Caracas. Revista de Investigación, 38(81), 69-88. En: http://www.scielo. org.ve/pdf/ri/v38n81/art04.pdf

8. CORTINA, A. \& MARTÍNEZ, E. (2008). Ética, España. Ediciones Akal.

9. GAUDLITZ, M. (2008). Reflexiones sobre los principios éticos en investigación biomédica en seres humanos. Departamento de Bioética y Humanidades Médicas, Vicepresidente Comité de Ética de Investigación en Seres Humanos. Facultad de Medicina, Universidad de Chile. En:http://www.scielo.cl/pdf/rcher/v24n2/art08.pdf

10. GEORGY, R. \& TOLLEFSEN, CH. (2012). Embrión: Una defencza de la vida humana. Ediciones Realp, S. A. Madrid.

11. MORALES, L. (S/A). Tendencias epis- temológicas en:http://es.scribd.com/ doc/55213706/TENDENCIAS-EPISTEMOLOGICAS.

12. MORÍN, E.(2004).La epistemología de la complejidad. Gazeta de antropología 2010.

13. OLIVE, L. (2007).Ciencia y la tecnología en la sociedad del conocimiento. México. Fondo de Cultura económica

14. PADRÓN, J. (2007). Tendencias Epistemológicas de la Investigación Científica en el Siglo XXI Cinta de Moebio 28: 1-28 Universidad Simón Rodríguez. Caracas, Venezuela. En:www.moebio.uchile.cl/28/padron.html1

15. PÉREZ, T. (2005). La perspectiva constructivista en la investigación social. Revista Tendencias \& Retos No 10:39-64 / Octubre. En: http://www.ts.ucr.ac.cr/binarios/revistas/ co/rev-co-tendencias-0010-03.pdf

16. RAMÓN, J. (2006). Ética. Historia y fundamentos, España. Albatros.

17. RAWLS, J. (1971). A theory of justice. The Belknap Press of Harvard University Press, EE.UU.Cambridge, Mass.

18. TAMAYO, M. (2004). El proceso de la investigación científica, México, Lemusa editores.

19. VALENZUELA, I. (S/A). Perspectivas epistemológicas. En:http://es.scribd.com/ doc/56419095/I-PERSPECTIVAS-EPISTEMOLOGICAS\#scribd.

20. VIEIRA, E. (2009). Principios epistemológicos a competencia informacional. IX congress ISko-Spain: New perspectives for the organization of knowledge. Valencia. En: file:///C:/Users/empresa/Downloads/ Dialnet-PrincipiosEpistemologicosACometenciaInformacionalD-2922007\%20(1).pdf. 